\title{
How to Improve the Convergent Argument Calculation
}

\author{
Alexander V. Tyaglo National University of \\ Internal Affairs (Ukraine)
}

\begin{abstract}
Quantitative apparatus for assessing strength of an argument was improved in the article. A probative generalisation and verification of the R. Yanal's algorithm to calculate convergent argument were proposed. The generalization obtained deals with both quantity (or number) and quality (or truth-values) of the argument's reasons.
\end{abstract}

Résumé: L'article présente l'approche quantitative à l'estimation de la force de l'argument. Il a été procédé à la généralisation prouvée et à la vérification de l'algoritme du calcul de l'argument convergent, proposé par R. Yanal. Cette généralisation concerne la quantité et la qualité des raisons de l'argument.

Keywords: informal logic, theory of argument, convergent argument, evaluation, Robert Yanal

\section{Introduction}

This article will pay attention to the contemporary tendency to elaborate a quantitative apparatus for assessing the degree of support that reasons lend to a conclusion, and hence the strength of an argument. This tendency or even program was well presented, for instance, by John Black. See Black $(1991,21)$. I will try to support this one for the sake of both research and pedagogic use.

There is no problem of finding argument diagrams, even in Beardsley's old book Practical Logic. The traditional approach to argument diagramming is quite friendly to intuition and simple in use. Perhaps owing to these properties, it seems hard to find principal changes in the essence of the approach or in diagramming techniques (see, e.g., Beardsley 1950, 18-26; Fisher 1988, 18-19; Moore and Parker 1998, 261-264; Browne 1998, 29-30; Thomas 1997, 49-63; Walton, C. 2000, TS 29-31). However, a few years ago Walton, D. (1996) published a monograph that provides relatively stricter grounds - directed graphs (digraphs) theoryfor the techniques. This innovation permits us to describe argument structure in a more sophisticated manner than does the traditional approach, and because it is able to solve a lot of widespread tasks, I will use this variant of argument diagramming further. 


\section{A short survey of traditional argument diagramming and evaluation}

In the simplest case, an argument consists of one reason (premise) and one conclusion. If we designate the reason by $\mathbf{R}$, the conclusion by $\mathbf{C}$, and the logical connection between them by a solid arrow directed from $\mathbf{R}$ to $\mathbf{C}$, then the simplest argument's structure will be represented by the diagram in Figure 1.

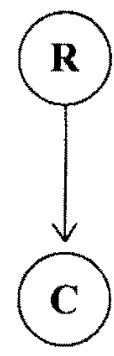

Figure 1

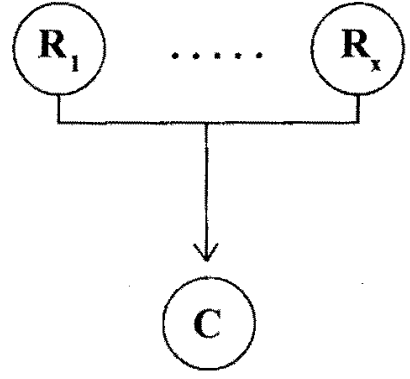

a

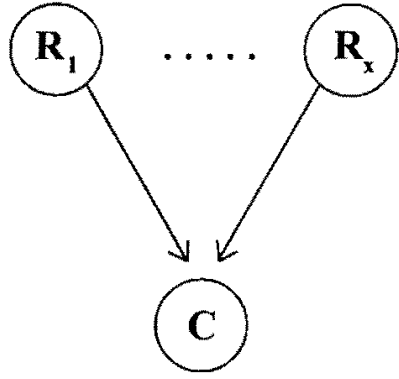

b

Figure 2

In more complex cases when the conclusion is supported by more than one reason, the arguments (and their diagrams) are divided into two main classes: those with mutually dependent reasons (such arguments are known as linked) (Figure 2a) and those with independent reasons (convergent arguments) (Figure $2 b)$.

There exist other logical divisions of arguments, for example "convergent divergent" (Figure 3a) and "one-stepimany-steps (serial)". When the conclusion of the first argument is a premise of the second one, etc., we have a serial argument (Figure 3b). 


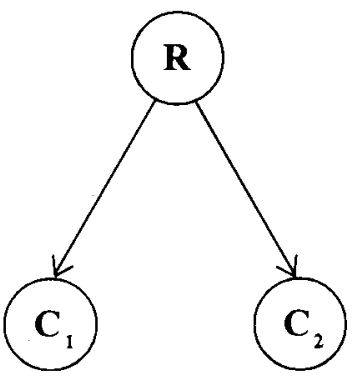

a

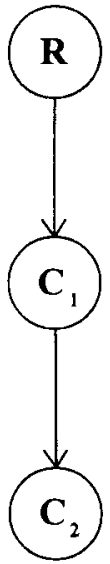

b

Figure 3

Diagramming techniques permit us not only to visualize an argument's structure, but also to reflect the strength of connection between reason(s) and conclusion. This approach provides a way to calculate an essentially quantitative characteristic of an argument--the probability of a conclusion being true if its reasons are acceptable.

About 10 years ago an American researcher, Robert Yanal, proposed a relevant algorithm to calculate the strength of a convergent argument. (Yanal 1988, 39-55; 1991, 137-144). He suggested dividing all arguments into strong, medium, weak and deductively valid depending on the degree of support that the reasons of an argument lend to their conclusion ("degree of validity").' The difference between such sorts of arguments is described quantitatively by a set of $\mathbf{p}\left(\mathbf{C} \mid \mathbf{R}_{\mathrm{x}}\right)$ - the probability that an independent $\mathbf{R}_{\mathbf{x}}$ lends to $\mathbf{C}$ or, in other words, the strength of connection between $\mathbf{R}_{\mathbf{x}}$ and $\mathbf{C}$.

In addition, Yanal pointed out that "we can evaluate the degree of validity of an argument without concerning ourselves with the truth of its premise(s)" (1988, 75). It becomes apparent that the degree of validity is indifferent to the truthvalues of the reasons or the conclusion and, therefore, does not characterize argument exhaustively.

For instance, the argument "My dog has fleas, therefore all dogs have fleas" was named weak and designated as $\mathbf{W}$. The argument "All dogs have fleas, therefore my dog has fleas" was named deductively valid and denominated as DV, etc. $(1988,83)$. In accordance with Yanal's proposition, the traditional diagrams are to be completed by signs $\mathbf{S}, \mathbf{M}, \mathbf{W}$ and $\mathbf{D V}$. 


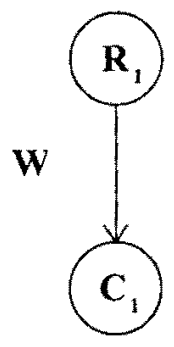

a

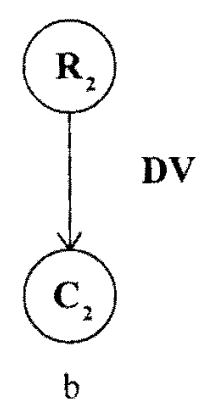

b

Figure 4

Yanal's algorithm to calculate the probability that the conclusion is true was substantiated by intuitive reasoning for a convergent argument with reasons $\mathbf{R}_{1}$ and $\mathbf{R}_{\mathbf{2}}$. Suppose a reason, $\mathbf{R}_{\mathbf{1}}$, is brought forward to support a conclusion, $\mathbf{C}$; and suppose that $\mathbf{R}_{1}$ lends 0.3 probability to $\mathbf{C}$. Analogously, independent $\mathbf{R}_{2}$ lends 0.4 probability to $\mathbf{C}$. Yanal writes:

Obviously, $\mathbf{C}$ is supported by two reasons whose probability together is greater than the probability of either one individually. But how much greater? It would be an error to simply add the probability of each reason $(0.3+0.4)$, thereby coming to the conclusion $\mathrm{C}$ is now supported with 0.7 probability. Here's why. If we know $\mathbf{R}_{1}$, then we have 0.3 certainty that $\mathbf{C}$ is true. Certainty can be thought as 1.0 probability. In other words, knowing that $\mathbf{C}$ is true on the basis of knowing that $\mathbf{R}_{1}$ is true is the same as knowing $\mathbf{C}$ with probability 0.3 times $1.0(=0.3)$. Therefore, merely knowing that $\mathbf{R}_{\mathbf{1}}$ is true leaves us with 0.7 ( $=1.0$ minus 0.3 ) of uncertainty. Now, when $\mathbf{R}_{2} \ldots$ is brought forward, we have decreased our uncertainty by 0.4 . In total, we know the conclusion with 0.3 (the probability of $\mathbf{R}_{1}$ ) plus 0.28 (the probability of $\mathbf{R}_{2}$ times the remaining "unknown" left over from 0.3 ), which equals $0.58 \ldots$ We shall call this the "ordinary" way of summing probabilities. (Yanal 1988,54.)

On the basis of this algorithm, Yanal proposed a criterion to distinguish arguments with independent and dependent reasons (1991, 140). But this criterion was substantively questioned (Conway 1991, 145-158).

It is necessary to point out that this algorithm de facto was supported by John Black (1991), but without reference to Yanal. Black simply postulated the rule without further test or even any illustration. Therefore for the sake of theoretical perfection and practical reliance, it is necessary to verify Yanal's algorithm in probative fashion. How it is possible to reach this goal?

\section{Generalization and verification of Yanal's algorithm}

To start with, let us define the next quantitative frames to differentiate connections between premise and conclusion: $\mathbf{p}\left(\mathbf{C} \mid \mathbf{R}_{\mathbf{X}}\right)$ (see Black 1991, 27). 
Weak connection

Medium connection

Strong connection

Deductively valid

Argument's connection

$$
\begin{aligned}
& 0 \leq \mathrm{p}\left(\mathrm{C} \mid \mathrm{R}_{\mathrm{x}}\right) \leq 1 / 2 \\
& 1 / 2<\mathrm{p}\left(\mathrm{C} \mid \mathrm{R}_{\mathrm{x}}\right) \leq 2 / 3 \\
& 2 / 3<\mathrm{p}\left(\mathrm{C} \mid \mathrm{R}_{\mathrm{x}}\right)<1
\end{aligned}
$$$$
\mathrm{p}\left(\mathrm{C} \mid \mathrm{R}_{\mathrm{x}}\right)=1
$$

Evaluation of the strength of argument with independent reasons is determined by two factors: the reasons' truth-values and the strength of connections between the reasons and conclusion reflected by $\mathbf{p}\left(\mathbf{C} \mid \mathbf{R}_{\mathbf{x}}\right)$.

Next, let us take into consideration the diagrams with true reasons only and denominate these reasons as $\mathbf{T}_{\mathbf{X}}{ }^{2}$ In cases with true reasons, the convergent argument's strength, i.e. the conclusion's probability of being true $\mathbf{P}\left(\ldots \mathbf{T}_{\mathrm{X}} \ldots\right)$, should be equal to the "ordinary sum" of $\mathbf{p}\left(\mathbf{C} \mid \mathbf{T}_{\mathbf{x}}\right)$. How can we explore Yanal's algorithm in a more probative way?

The questioned algorithm is clearly represented by formula $(1):^{3}$

$$
\mathrm{p}\left(\mathrm{C} \mid \mathrm{T}_{1}, \mathrm{~T}_{2}\right)=\mathrm{p}\left(\mathrm{C} \mid \mathrm{T}_{1}\right)+\left\{1-\mathrm{p}\left(\mathrm{C} \mid \mathrm{T}_{1}\right)\right\} \mathrm{p}\left(\mathrm{C} \mid \mathrm{T}_{2}\right)
$$

It is reasonable to generalize (1) for an unlimited number of reasons (or premises) $\mathbf{T}_{\mathbf{X}}, 1 \leq \mathbf{X} \leq \infty$. In this way we will arrive at (2).

$$
\begin{aligned}
& \mathrm{p}\left(\mathbf{C} \mid \mathbf{T}_{1} \mathbf{T}_{2}\right)=\mathrm{p}\left(\mathbf{C} \mid \mathbf{T}_{1}\right)+\left\{\mathbf{1}-\mathbf{p}\left(\mathbf{C} \mid \mathbf{T}_{1}\right)\right\} \mathrm{p}\left(\mathbf{C} \mid \mathbf{T}_{2}\right) \\
& \mathbf{p}\left(\mathbf{C} \mid \mathbf{T}_{1}, \mathbf{T}_{2}, \mathbf{T}_{3}\right)=\mathbf{p}\left(\mathbf{C} \mid \mathbf{T}_{1}\right)+\left\{\mathbf{1}-\mathbf{p}\left(\mathbf{C} \mid \mathbf{T}_{1}\right)\right\} \mathbf{p}\left(\mathbf{C} \mid \mathbf{T}_{2}\right)+\left\{\mathbf{1}-\mathbf{p}\left(\mathbf{C} \mid \mathbf{T}_{1}\right)-\left[1-\mathbf{p}\left(\mathbf{C} \mid \mathbf{T}_{1}\right)\right] \times\right. \\
& \left.\times \mathbf{p}\left(\mathbf{C} \mid \mathbf{T}_{2}\right)\right\} \times \mathbf{p}\left(\mathbf{C} \mid \mathbf{T}_{3}\right)= \\
& =\mathbf{p}\left(\mathbf{C} \mid \mathbf{T}_{1}, \mathbf{T}_{2}\right)+\left\{\mathbf{1 - p}\left(\mathbf{C} \mid \mathbf{T}_{1}, \mathbf{T}_{2}\right)\right\} \mathbf{p}\left(\mathbf{C} \mid \mathbf{T}_{\mathfrak{y}}\right) \\
& p\left(C \mid T_{1}, \ldots T_{x}\right)=p\left(C \mid T_{1}, \ldots T_{X-1}\right)+\left\{1-p\left(C \mid T_{1}, \ldots T_{X-1}\right)\right\} p\left(C \mid T_{X}\right)
\end{aligned}
$$

In order to verify (1) and each of its generalizations, let us introduce a set of natural essential conditions $\mathbf{C 1}$ - $\mathbf{C 4}$.

The first condition $\mathbf{C 1}$ presupposes that the correctly calculated probability $\mathbf{p}\left(\mathbf{C} \mid \ldots \mathbf{R}_{\mathrm{x}} \ldots\right)$ is to lie between 0 and $1 .{ }^{4} \mathbf{C} 2$ demands that this probability does not depend on a calculating process (in other words, it is possible to include any $\mathbf{p}\left(\mathbf{C} \mid \mathbf{R}_{\mathbf{x}}\right)$ into the "ordinary sum" in an arbitrary order). $\mathbf{C} \mathbf{3}$ demands: if a conclusion has at least one sufficient reason $\mathbf{R}_{\mathrm{x}}$ and, therefore, there is a probability $\mathbf{p}\left(\mathbf{C} \mid \mathbf{R}_{\mathrm{x}}\right)=1$, then all others are not able to change anything in $\mathbf{p}\left(\mathbf{C} \mid \ldots \mathbf{R}_{\mathrm{x}} \ldots\right)=1$. And condition $\mathbf{C 4}$ demands: if a conclusion has irrelevant reason(s), it does not influence one's probability.

In the special case with true premises, $\mathbf{C 1 - C 4}$ will be modified to next $\mathbf{C 1}^{\prime}$ C4'. 


\begin{tabular}{|c|c|}
\hline $\begin{array}{l}0 \leq p\left(C \mid \ldots T_{x} \ldots\right) \leq 1, \\
p\left(C \mid \ldots T_{x}, T_{x+1} \ldots\right)= \\
=p\left(C \mid \ldots T_{x+1}, T_{x} \ldots\right) \text { etc., } \\
\text { if } p\left(C \mid T_{x}\right)=1,1<X \leq \infty, \\
\text { then } p\left(C \mid T_{1} \ldots T_{x} \ldots\right)=1, \\
\text { if } p\left(C \mid T_{x}\right)=0,1<X \leq \infty, \\
\text { then } p\left(C \mid \ldots T_{x-1}, T_{x}, T_{x+1} \ldots\right)= \\
=p\left(C \mid \ldots T_{x-1}, T_{x+1} \ldots\right)\end{array}$ & $\begin{array}{l}\mathrm{C} 2^{\prime} \\
\mathrm{C} 3^{\prime}\end{array}$ \\
\hline
\end{tabular}

Now, let us test if (1) meets $\mathbf{C 1}$ '-C4'.

If the initial conditions were stated correctly, then $0 \leq \mathbf{p}\left(\mathbf{C} \mid \mathbf{T}_{1}\right) \leq 1$ and $0 \leq$ $\mathbf{p}\left(\mathbf{C} \mid \mathbf{T}_{2}\right) \leq 1$, therefore $\mathbf{p}\left(\mathbf{C} \mid \mathbf{T}_{1}, \mathbf{T}_{2}\right) \geq 0$. On the other hand: $1-\mathbf{p}\left(\mathbf{C} \mid \mathbf{T}_{1}\right) \geq[1-$ $\left.\mathbf{p}\left(\mathbf{C} \mid \mathbf{T}_{1}\right)\right] \mathbf{p}\left(\mathbf{C} \mid \mathbf{T}_{2}\right)$. Hence, $\mathbf{p}\left(\mathbf{C} \mid \mathbf{T}_{1}\right)+\left[1-\mathbf{p}\left(\mathbf{C} \mid \mathbf{T}_{1}\right)\right] \times \mathbf{p}\left(\mathbf{C} \mid \mathbf{T}_{2}\right) \leq 1$. Thus, $\mathbf{C} \mathbf{1}^{\prime}$ is met.

$\mathbf{C 2}{ }^{\prime}$ is met because (1) includes $\mathbf{p}\left(\mathbf{C} \mid \mathbf{T}_{1}\right)$ and $\mathbf{p}\left(\mathbf{C} \mid \mathbf{T}_{2}\right)$ symmetrically.

Under condition $\mathbf{p}\left(\mathbf{C} \mid \mathbf{T}_{1}\right)=1, \mathbf{C 3}^{r}$ is met because multiplier near $\mathbf{p}\left(\mathbf{C} \mid \mathbf{T}_{2}\right)$ equals 0 and this component's share is nullified. An analogous conclusion for $\mathbf{p}\left(\mathbf{C} \mid \mathbf{T}_{2}\right)=1$ will be drawn if we take into account $\mathbf{C 2}^{\prime}$.

If $\mathbf{p}\left(\mathbf{C} \mid \mathbf{T}_{1}\right)=0$, then $\mathbf{C} \mathbf{4}^{\prime}$ is obviously met; owing to $\mathbf{C} \mathbf{2}^{\prime}$ we arrive at analogous conclusion in the case with $\mathbf{p}\left(\mathbf{C} \mid \mathbf{T}_{2}\right)=0$.

There is no problem in proving that generalization (2) meets $\mathbf{C 1}^{\prime}-\mathbf{C 4}^{\prime}$ as well, but in analytical form it is a task for the most curious people. I will simply verify (2) by a concrete example.

For the diagram on Figure 5, let us suppose: $\mathbf{p}\left(\mathbf{C} \mid \mathbf{T}_{1}\right)=1 / 2, \mathbf{p}\left(\mathbf{C} \mid \mathbf{T}_{2}\right)=2 / 3$, $\mathbf{p}\left(\mathbf{C} \mid \mathbf{T}_{3}\right)=0, \mathbf{p}\left(\mathbf{C} \mid \mathbf{T}_{4}\right)=1$.

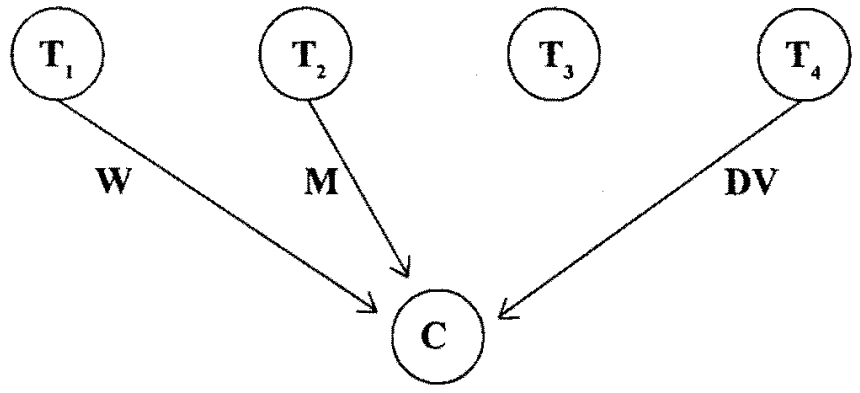

Figure 5

Step by step from (2), we will figure out $\mathbf{p}\left(\mathbf{C} \mid \mathbf{T}_{1}, \mathbf{T}_{2}, \mathbf{T}_{3}, \mathbf{T}_{4}\right)$.

$$
\begin{aligned}
& \mathbf{p}\left(\mathbf{C} \mid \mathbf{T}_{1}, \mathbf{T}_{2}\right)=1 / 2+(1-1 / 2) \times 2 / 3=5 / 6, \\
& \mathbf{p}\left(\mathbf{C} \mid \mathbf{T}_{1}, \mathbf{T}_{2}, \mathbf{T}_{3}\right)=5 / 6+(1-5 / 6) \times 0=5 / 6, \\
& \mathbf{p}\left(\mathbf{C} \mid \mathbf{T}_{1}, \mathbf{T}_{2}, \mathbf{T}_{3}, \mathbf{T}_{4}\right)=5 / 6+(1-5 / 6) \times 1=1
\end{aligned}
$$


This result meets $\mathbf{C 1}^{\prime}, \mathbf{C 3}^{\prime}$ and $\mathbf{C 4}^{\prime}$ clearly. Repeat the exercise for, e.g., $\mathbf{p}\left(\mathbf{C} \mid \mathrm{T}_{2}, \mathrm{~T}_{4}, \mathrm{~T}_{1}, \mathrm{~T}_{3}\right)$.

$$
\begin{aligned}
& \mathbf{p}\left(\mathbf{C} \mid \mathbf{T}_{2}, \mathbf{T}_{4}\right)=2 / 3+(1-2 / 3) \times 1=1, \\
& \mathbf{p}\left(\mathbf{C} \mid \mathbf{T}_{2}, \mathbf{T}_{4}, \mathbf{T}_{1}\right)=1+(1-1) \times 1 / 2=1, \\
& \mathbf{p}\left(\mathbf{C} \mid \mathbf{T}_{2}, \mathbf{T}_{4}, \mathbf{T}_{1}, \mathbf{T}_{3}\right)=1+(1-1) \times 0=1 .
\end{aligned}
$$

Hence, $\mathbf{p}\left(\mathbf{C} \mid \mathbf{T}_{1}, \mathbf{T}_{2}, \mathbf{T}_{3}, \mathbf{T}_{4}\right)=\mathbf{p}\left(\mathbf{C} \mid \mathbf{T}_{2}, \mathbf{T}_{4}, \mathbf{T}_{1}, \mathbf{T}_{3}\right)$ in accordance with $\mathbf{C} 2 \xi !$

Further generalization of (1) and (2) permits us to take into consideration an argument with unknown true-values of reasons. More exactly, in this case we know accurately only $\mathbf{P}\left(\mathbf{H}_{\mathrm{x}}\right)$-the probability that a reason $\mathbf{H}_{\mathrm{x}}$ is true. In terms of Black's paper this is "the assigned probability" (see Black 1991, 27, 30). Let us name such a set of reasons and arguments as hypothetical.

It is impossible to identify the hypothetical argument's strength $\mathbf{P}\left(\mathbf{C} \mid \mathbf{H}_{1} \& \ldots \mathbf{H}_{\mathbf{x}} \& \ldots\right)$, i.e. the probability of the reasons and conclusion being true at one time, with the "ordinary sum" $\mathbf{p}\left(\mathbf{C} \mid \ldots \mathbf{R}_{\mathbf{x}} \ldots\right)$ as it was possible in the situation with a true premises argument. Indeed, in general, the argument's strength depends both on reason-conclusion connections $\mathbf{p}\left(\mathbf{C} \mid \mathbf{H}_{\mathbf{x}}\right)$ and the probability of a reason being true $\mathbf{P}\left(\mathbf{H}_{\mathbf{x}}\right)$.

In accordance with the elementary theory of probability that we have for the conditional probability $\mathbf{p}(\mathbf{C} \mid \mathbf{H})=\mathbf{P}(\mathbf{C \& H}) / \mathbf{P}(\mathbf{H})$, we arrive at (3):

$$
\mathbf{P}(\mathbf{C \& H})=\mathbf{P}(\mathbf{H}) \mathbf{p}(\mathbf{C} \mid \mathbf{H})
$$

For instance, if $\mathbf{P}\left(\mathbf{H}_{1}\right)=0.7$ and $\mathbf{p}\left(\mathbf{C} \mid \mathbf{H}_{1}\right)=0.7$, then the probability of the argument's reason and conclusion being true one time will be 0.49 . Suppose $\mathbf{p}\left(\mathbf{C} \mid \mathbf{H}_{2}\right)$ $=1$, but $\mathbf{P}\left(\mathbf{H}_{2}\right)=0.1$. Then $\mathbf{P}\left(\mathbf{C} \& \mathbf{H}_{2}\right)=0.1$, and we get a deductively valid but practically unsound argument.

On the basis of the last generalization, it is possible to calculate a serial argument that might include hypothetical premises. For instance, if $\mathbf{p}(\mathbf{H} \mid \mathbf{T})=0.7, \mathbf{p}(\mathbf{C} \mid \mathbf{H})$ $=0.7$, then we will get the result stated above: $\mathbf{P}(\mathbf{C \& H} \& \mathbf{T})=\mathbf{P}(\mathbf{T}) \mathbf{p}(\mathbf{H} \mid \mathbf{T}) \times$ $\mathbf{p}(\mathbf{C} \mid \mathbf{H})=1 \times \mathbf{p}(\mathbf{H} \mid \mathbf{T}) \mathbf{p}(\mathbf{C} \mid \mathbf{H})=0.49$ (Figure 6).

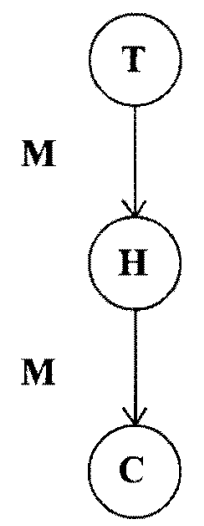

Figure 6 
It is not too difficult to introduce a formula (4) for a convergent argument with two hypothetical reasons.

$\mathbf{P}\left(\mathrm{C} \& \mathrm{H}_{1} \& \mathrm{H}_{2}\right)=\mathbf{P}\left(\mathrm{H}_{1}\right) \mathbf{p}\left(\mathbf{C} \mid \mathrm{H}_{1}\right)+\left[\mathbf{1}-\mathbf{P}\left(\mathrm{H}_{1}\right) \mathbf{p}\left(\mathbf{C} \mid \mathrm{H}_{1}\right)\right] \mathbf{P}\left(\mathrm{H}_{2}\right) \mathbf{p}\left(\mathbf{C} \mid \mathbf{H}_{2}\right)$

Let us check whether (4) meets the principal conditions $\mathrm{C} 1-\mathrm{C} 2$ by a particular example relevant to Figure 7 .

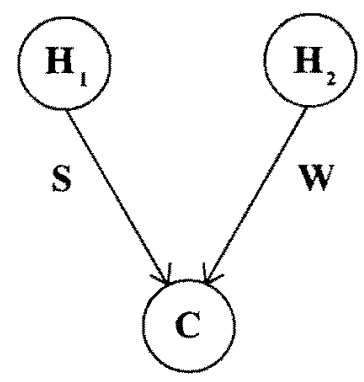

Figure 7

The probability that the statement "The brake in a repaired car is out of order" is true, equals $P\left(H_{1}\right)=0.0001$. The probability that statement "The car's motor is out of order" is true, equals $\mathrm{P}\left(\mathrm{H}_{2}\right)=0.01$. When the car has malfunctioning brakes then the probability of a quick fender-bender is $\mathrm{p}\left(\mathrm{C} \mid \mathrm{H}_{1}\right)=0.9$; with malfunctioning motor it equals $\mathrm{p}\left(\mathrm{C} \mid \mathrm{H}_{2}\right)=0.25$. How much is the probability that the forecast about the car's imminent crash will be true?

$\mathbf{P}\left(\mathbf{C} \& \mathbf{H}_{1} \& \mathbf{H}_{2}\right)=\mathbf{P}\left(\mathbf{H}_{1}\right) \mathbf{p}\left(\mathbf{C} \mid \mathbf{H}_{1}\right)+\left[1-\mathbf{P}\left(\mathbf{H}_{1}\right) \mathbf{p}\left(\mathbf{C} \mid \mathbf{H}_{1}\right)\right] \mathbf{P}\left(\mathbf{H}_{2}\right) \mathbf{p}\left(\mathbf{C}_{2} \mathbf{H}_{2}\right)=$ $0.0001 \times 0.9 \times(1-0.00009) \times 0.01 \times 0.25=0.002589775$,

$\mathbf{P}\left(\mathbf{C} \& \mathbf{H}_{2} \& \mathbf{H}_{1}\right)=0.01 \times 0.25+(1-0.0025) \times 0.0001 \times 0.9=0.002589775$.

So, $\mathbf{C 1}$ and $\mathbf{C 2}$ are met.

Another case is possible in which probability of a motor defect is excluded, i.e. $\mathbf{P}\left(\neg \mathbf{H}_{2}\right)=1$ and $\mathbf{p}\left(\mathbf{C} \mid \neg \mathbf{H}_{2}\right)=0$ : in this case we calculate the possibility of a crash, caused by a malfunctioning brake only $\mathbf{P}\left(\mathbf{C} \& \mathbf{H}_{1} \& \neg \mathbf{H}_{2}\right)$.

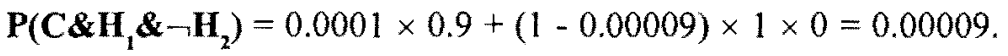

Thus, (4) meets $\mathbf{C 4}$ as well. Checking the $\mathbf{C} 3$ condition for this case and generalizing from (4) does not create any significant problems. However, because of the technical complexity I will avoid these exercises now.

Let us instead take into consideration a case with zero probability that a reason is true. The meaning of this case is quite clear: a reason $\mathbf{F}_{\mathbf{x}}$ is false; i.e., it does not correspond to reality.

Examine, e.g., the next diagram with $\mathbf{P}(\mathbf{H})=0.5, \mathbf{p}(\mathbf{C} \mid \mathbf{H})=0.6, \mathbf{P}(\mathbf{F})=0$, $\mathbf{p}(\mathbf{C} \mid \mathbf{F})=1$. 


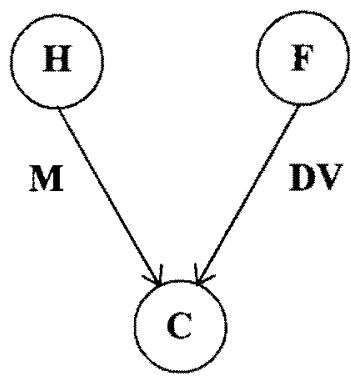

Figure 8

$\mathbf{P}(\mathbf{C \& H})=\mathbf{P}(\mathbf{H}) \mathbf{p}(\mathbf{C} \mid \mathbf{H})=0.3$.

$\mathbf{P}(\mathbf{C \& H \& F})=\mathbf{P}(\mathbf{H}) \mathbf{p}(\mathrm{C} \mid \mathbf{H})+[1-\mathbf{P}(\mathbf{H})(\mathbf{C} \mid \mathbf{H})] \mathbf{P}(\mathbf{F}) \mathbf{p}(\mathbf{C} \mid \mathbf{F})=\mathbf{P}(\mathbf{H}) \mathbf{p}(\mathbf{C} \mid \mathbf{H})=$ 0.3 .

Hence, $\mathbf{P}(\mathbf{C} \& \mathbf{H} \& \mathbf{F})=\mathbf{P}(\mathbf{C} \& \mathbf{H})$. Even though spite of the connection between $\mathbf{F}$ and $\mathbf{C}$ is $\mathbf{D V}$, the false reason must not lend any real support to the conclusion. This naturally presupposed result verifies the questioned algorithm once more.

\section{Brief conclusion}

In this article a probative generalization and verification of Yanal's algorithm to calculate a convergent argument have both been proposed. The results obtained deal with both quantity (or number) and quality (or truth-values) of the argument's reason(s). Generally speaking, the quantitative apparatus for assessing the strength of the arguments was improved.

It is particularly noteworthy that the generalization proposed has the same limits as Yanal's basic algorithm. First, the initial assignment of accurate numerical values both to reason-conclusion connections $\mathbf{p}\left(\mathbf{C} \mid \mathbf{H}_{\mathrm{x}}\right)$ and the probability of reason being true $\mathbf{P}\left(\mathbf{H}_{\mathbf{x}}\right)$ in many practical cases is intuitive. Therefore it might be subjective and controversial (cf., Black 1991, 29; and Walton 1996, 128-129). Second, as Professor Wayne Grennan has hinted to me, the formula (2) and (4) are only approximations to accurate formulae. Mathematically correct formulae might be derived based on a relevant J. M. Keynes' equation. But in a regular case this equation includes additional unknown variables and so isn't solvable. ${ }^{5}$ In order to get any numerical solution we must return to approximate Yanal's algorithm and its different modifications. Correspondence between the approximations and relevant accurate solutions constitutes an open research problem, and I intend to study it further.

The results obtained do not exhaust all the possibilities for improving traditional argument evaluation. It seems reasonable, for example, to reflect in an argument diagram and calculation not only pro but contra reasons as well. ${ }^{6}$ The issue demands further investigations both scientifically and pedagogically. 


\section{Acknowledgements}

The core idea of the paper arose through discussions with Dr. Edward F. McClennen, Dr. M. Neil Brown and Dr. Stuart M. Keeley during my research visit to the Bowling Green State University in 1998. This visit was supported by the International Research and Exchange Board(IREX), with funds provided by the United States Informational Agency. I would like to thank the referee of an earlier version of this paper, Dr. Wayne Grennan, for his constructive criticism and remarks.

\section{Notes}

${ }^{\prime}$ An analogous degree of support scale was introduced in Thomas (137-138, etc.). Specifically important in this case is the explicit connection between degrees of validity and the decision making based on quantitative evaluation.

${ }^{2}$ Let us denote false reasons as $\mathbf{F}_{\mathbf{X}}$ and reasons with unknown truth values as $\mathbf{H}_{\mathbf{x}}$. A set of all possible reasons will be $\mathbf{R}=\mathbf{T} \cup \mathbf{H} \cup \mathbf{F}$.

${ }^{3}$ An equivalent formula was introduced in Black (24).

"This most obvious condition appeared in the Black's article.

'Grennan refers to the Keynes' formula for a two-premises convergent argument:

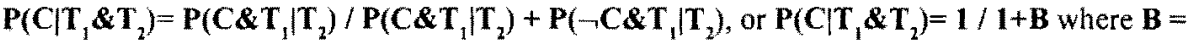
$=\mathbf{P}\left(\neg \mathbf{C \& T _ { 1 }} \mid \mathbf{T}_{2}\right) / \mathbf{P}\left(\mathbf{C \& T _ { 1 }} \mid \mathbf{T}_{2}\right)$ (see Keynes 1973, 165-166). In addition to the sought value

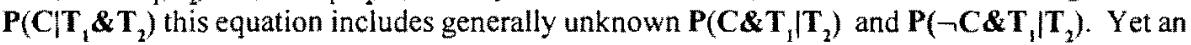
equation with two or more independent unknown variables is unsolvable.

${ }^{6}$ See, e.g., the intention in Moore and Parker (264), Thomas (386-388) and Black (25).

\section{References}

Beardsley, M. C. (1950). Practical Logic, Englewood Cliffs, NJ: Prentice Hall, Inc.

Black, J. (1991). "Quantifying Support," Informal Logic 13: 21.

Browne, M. N. and S. M. Keeley (1998). Asking the Right Questions, A Guide to Critical Thinking, $5^{\text {th }}$ ed. Englewood Cliffs, NJ: Prentice Hall, Inc.

Conway, D. P. (1991). "On the Distinction between Convergent and Linked Arguments," Informal Logic 13: 145.

Fisher, A. (1988). The Logic of Real Argument, Cambridge: Cambridge University Press.

Keynes, J. M. (1973). The Collected Writings of John Maynard Keynes. Vol. VIII. A Treatise on Probability. Cambridge: Cambridge University Press.

Moore, B .N. and R. Parker (1998). Critical Thinking, $5^{\text {th }}$ ed. Mountain View, CA: Mayfield Publishing Company.

Thomas, S. N. (1997). Practical Reasoning in Natural Language, $4^{\text {th }}$ ed. Upper Saddle River, NJ: Prentice-Hall, Inc.

Walton, D. (1996). Argument Structure: A Pragmatic Theory. Toronto: University of Toronto Press.

Walton, C. (2000). "Six Steps in Critical Thinking," Informal Logic, 20: TS 29. 
Yanal, R. J. (1988). Basic Logic. St.Paul: West Publishing Company.

Yanal, R. J. (1991). "Dependent and Independent Reasons," Informal Logic 12: 137.

Alexander V. Tyaglo Depariment of Philosophy and Political Science National University of Internal Affairs 27 Prosp. 50-richya SRSR Kharkiv, 61080 Ukraine alext@adm.univd.kharkov.ua 